\title{
The use of high frequency skin ultrasound in non-melanoma skin cancer
}

\author{
Artur Bezugly, MD, PhD, Assoc. Prof.! Agata Rembielak, BSc, MD, PhD, MA, MRCR² \\ INon-invasive Skin Diagnostics Laboratory ANTA-Med, Dermatology Department, Academy of Postgraduate under the Federal State \\ Budgetary Unit "Federal Scientific and Clinical Center for Specialized Medical Assistance and Medical Technologies of the Federal Medical \\ Biological Agency", Moscow, Russia, 2The Christie NHS Foundation Trust and The University of Manchester, Manchester, UK
}

\begin{abstract}
Ultrasound (US) or sonography is the most common diagnostic non-invasive imaging method, which is estimated to account for more than a third of all diagnostic procedures used in medical practice. Traditionally, US frequencies used in diagnostic radiology range from $1 \mathrm{MHz}$ to approximately $16 \mathrm{MHz}$. Visualization of skin morphology requires US frequencies from 20 to $100 \mathrm{MHz}$, and is called a "high frequency ultrasound" (HFUS). HFUS has been explored in normal skin and in various skin pathologies. It has been found useful in differentiation diagnosis of infiltrative (perivascular) and exudative inflammation, hypertrophic or normotrophic scars, keloids, and in skin atrophy. Moreover, it has been used to support diagnosis and management of skin tumors. In skin oncology, HFUS can provide essential information on tumors' size and margins, particularly the depth of invasion. Such information are crucial in deciding on the form of treatment (e.g., photodynamic therapy, radiotherapy, brachytherapy), computed tomography (CT)-based planning treatment (e.g., superficial brachytherapy), and in skin monitoring after treatment, in addition to clinical examination.

Key words: ultrasound, non-melanoma skin cancer, high frequency skin ultrasound.

\section{Ultrasound examination and high frequency ultrasound}

Non-invasive imaging of human organs and tissues is one of the most vital trends in medical diagnostics [1]. Different diagnostics methods, including X-ray computed tomography, magnetic resonance imaging, and diagnostic ultrasound are quickly evolving and improving. Ultrasound (US) or sonography is the most common non-invasive diagnostic method. In 2000-2011, the number of ultrasound imaging procedures increased more than a ten-fold $[1,2]$. It is relatively inexpensive, portable, and does not involve ionizing radiation.

Traditionally, US frequencies used in diagnostic radiology range from $1 \mathrm{MHz}$ to approximately $16 \mathrm{MHz}$. The traditional scanning probes with a frequency of $1-3.5 \mathrm{MHz}$ are used for deep-seated structures abdomen and obstetric imaging, $5 \mathrm{MHz}$ in breast and pelvis imaging, and $7.5-16 \mathrm{MHz}$ in breast, thyroid, superficial veins, superficial masses, and musculoskeletal imaging. The resolution of sonography strictly depends on the frequency. The higher the frequency of US, the better resolution achieved. Visualization of skin layers, such as hypodermis, especially dermis and epidermis, requires much higher frequencies, since skin morphological structures have a small dimension; the thickness of epidermis on the face and body is $70-120 \mu$, and this of palmar and plantar skin is $220-600 \mu[3,4]$, whereas the thickness of dermis is $0.7-4.5 \mathrm{~mm}[3,5]$. High frequency ultrasound (HFUS) uses frequencies in the range of 20 to $100 \mathrm{MHz}$ [6]. The resolution of this method is very high, varying from 80 to $16 \mu$ (10-6 m), allowing to visualize objects measuring approximately $0.08 \mathrm{~mm}$ up to $0.016 \mathrm{~mm}$. The HFUS physical principle is based on the fact that ultrasonic wave reflects the boundaries between structural parts of biologic tissue (e.g., fibers, cells, blood, lymphatic vessels), with different acoustic properties, such as acoustic impedance and ultrasound speed in the tissue.

US sensitivity is very high and allows for tissue differentiating with only a $0.1 \%$ density difference. Due to high heterogeneity of the epidermis, dermis, and subcutaneous fat structural components, HFUS scanning produces images matching the real skin morphology.

HFUS probe contains piezoelectric crystal, which emits ultrasonic waves and receives ultrasonic waves reflected from biological tissues. The principles of HFUS are presented in Figure 1. As the transducer in Figure 1 moves over the skin, it works in the pulse-echo regime. The red vertical line in Figure 2 corresponds to the ultrasound beam penetration in the skin. As the US wave beam penetrates deep into the 


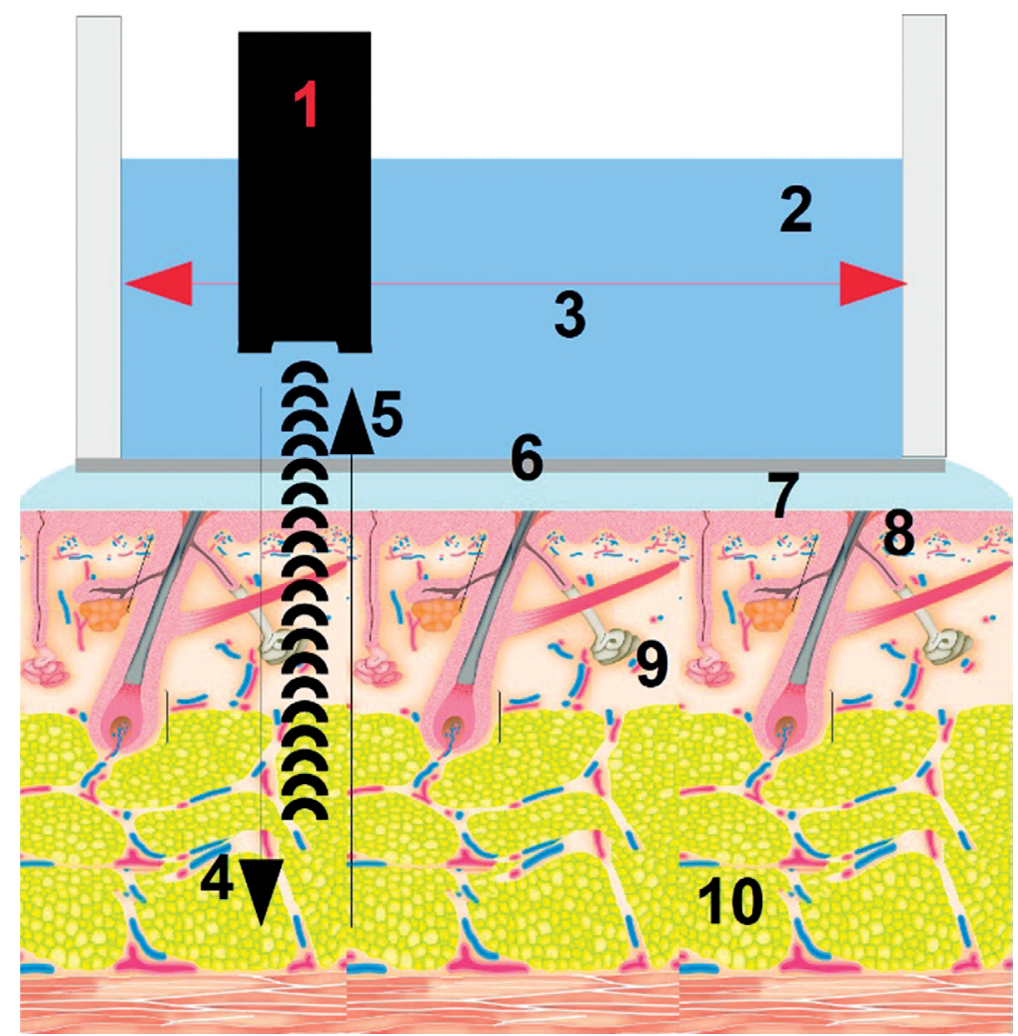

Fig. 1. HFUS skin scanning principles. 1. Piezoelectric probe, 2. Water in the applicator tip, 3. Direction of the ultrasound crystal movement, 4. Penetrating ultrasound wave, 5. Reflected ultrasound waves, 6. Membrane on the applicator tip, 7. Ultrasound contact gel, 8. Epidermis, 9. Dermis, 10. Subcutaneous fat

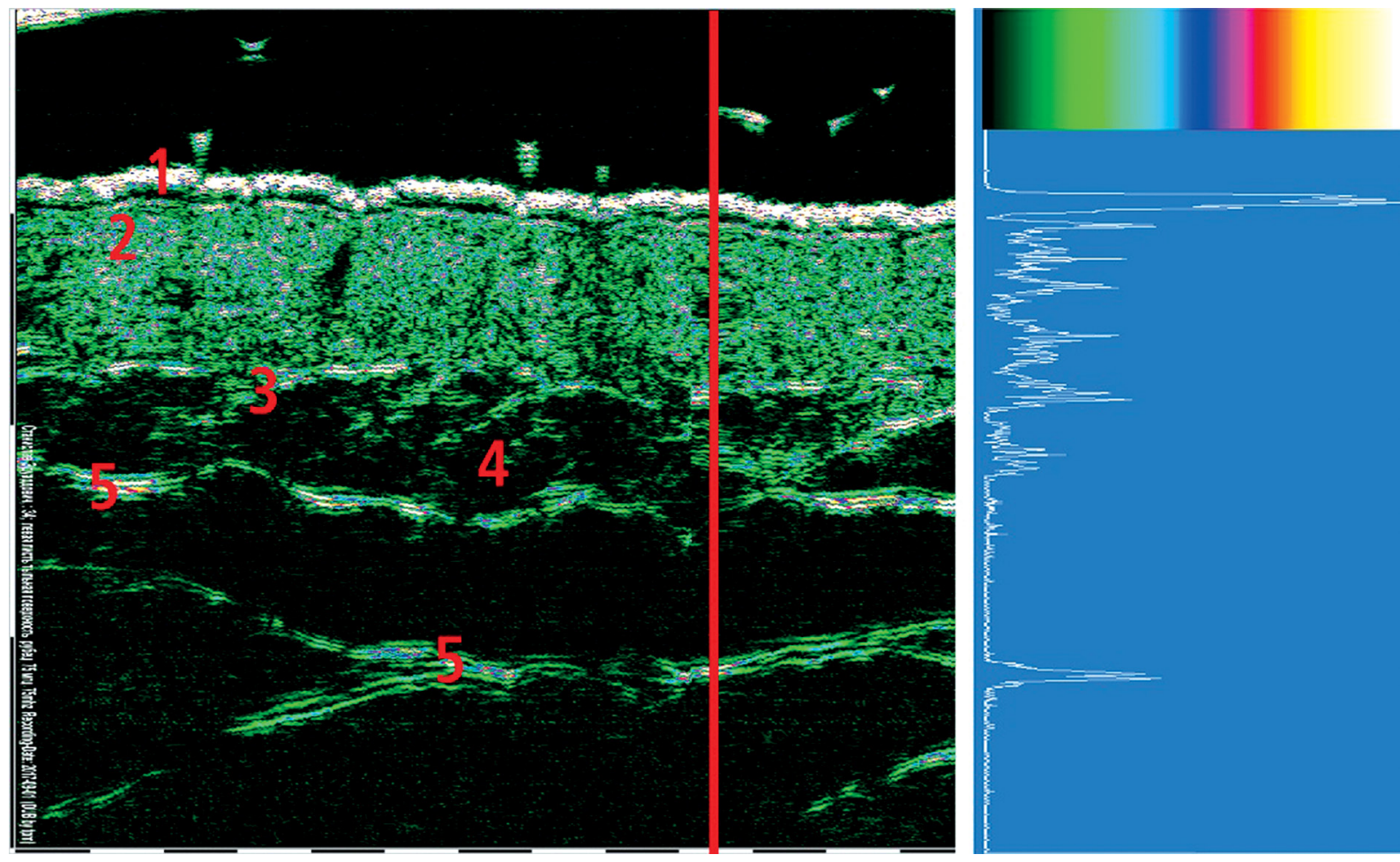

Fig. 2. Two-dimensional $75 \mathrm{MHz}$ HFUS scan and one-dimensional A-scan. Red vertical line corresponds to the direction of penetration and the reflection of ultrasonic wave. 1. Epidermis, 2. Dermis, 3. Lower dermis, with border between dermis and subcutaneous fat, 4 . Subcutaneous fat, 5 . Superficial fascia 
tissue, part of the ultrasonic waves is reflected from structural elements of the tissue and sent back to the transducer. The amplitude of each reflected signal is registered by the transducer and stored within the system. This amplitude curve is the record of signal reflected from the structural elements of tissue located at different depths, and is called "amplitude" or "A-scan". The white amplitude curve on the blue background seen in Figure 2 is an example of A-scan. The strength of reflected signal varies significantly for different structural elements of the skin.

Figure 2 presents intact dorsal palmar skin $75 \mathrm{MHz}$ scan of a 34-year-old healthy person. The strongest amplitude is recorded in the epidermis because it contains elements with the highest acoustic impedance heterogeneity: keratinized scales of the stratum corneum, lipids in the intercellular spaces, and the spinose and basal layers cells. The thin sub-epidermal anechoic band is most probably a kind of SLEB (sub-epidermal low echogenicity band). SLEB, also known as "sub-epidermal non-echogenic band" (SENEB), which is usually visualized on UV damaged skin, or in patients with inflammatory skin diseases [6]. The dermis contains elements of various density, such as collagen and elastin fibers, which are combined into bundles, glycosaminoglycans, cells, blood and lymph vessels, and nerve fibers. Therefore, the amplitude of reflected signals fluctuates between low and medium values. The main elements of fatty tissue lipocytes are $90 \%$ filled with fatty vacuoles, which determines the uniformity of acoustic density and results in very low amplitude of the

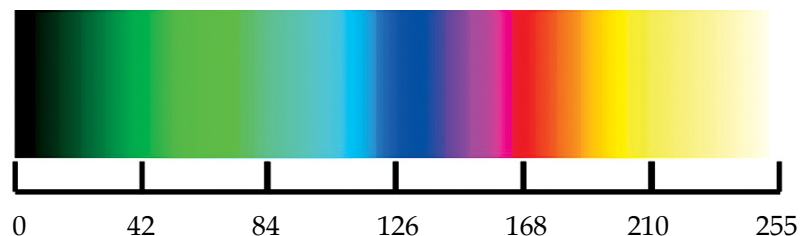

Fig. 3. Color scale used to convert amplitude values into color pixels. Level 0 is displayed in black color, level 42 in green, 84 in light green, 126 in blue, 168 in red, 210 yellow, and level 255 in white color

reflected signal. Connective tissue fascia is much denser than adipose and muscle tissue, and reflects ultrasound waves well, so the amplitude of reflected signal is medium and high.

The reflected signal amplitude is then converted into a pixel color in two-dimensional image. As it can be seen in Figure 2, there is a color scale above the A-scan diagram used to convert amplitude values to adequate pixel color. During digital conversion process, the amplitude values are separated at 256 levels, ranging from 0 to 255 . Zero amplitude is displayed in black color, low values are dark green and green, medium values are blue, purple, and pink, and high amplitude values are presented in red, orange, and yellow colors. The highest amplitude at 255 level is shown as white color. Figure 3 shows an example of conversion from amplitude value to color presentation.

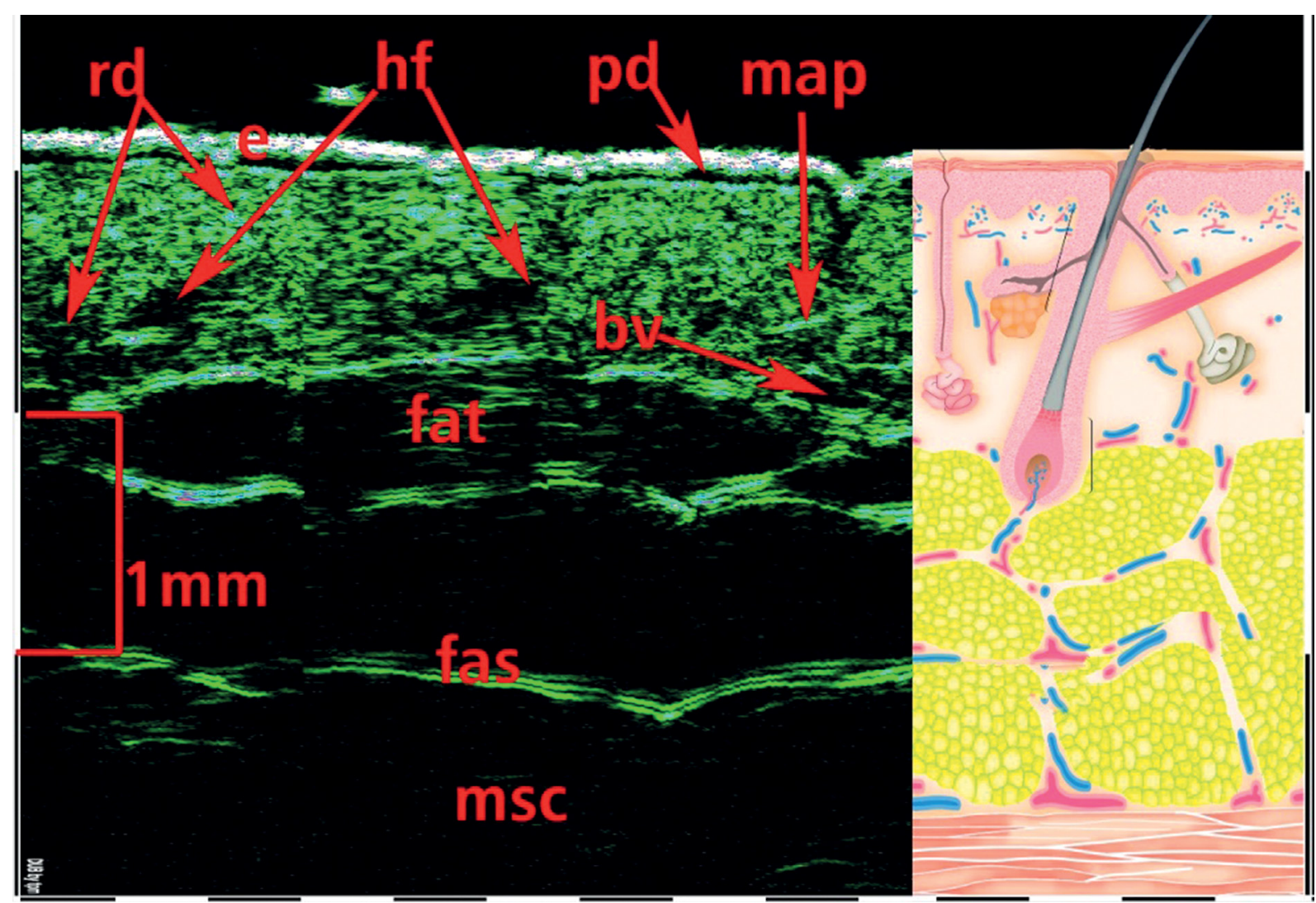

Fig. 4. $75 \mathrm{MHz}$ HFUS image of normal skin taken from the inner forearm. e - epidermis, pd - papillary dermis, rd - reticular dermis, bv - blood vessels, fat - subcutaneous fat tissue, fas - fascia, msc - muscle, hf - hair follicle, map - musculus arrector pili 
Depending on the type of US system, the device can store from 128 up to 386 A-scans used to build twodimensional vertical slice-cut skin image. The penetration depth depends on the US frequency. For example, $22 \mathrm{MHz}$ US wave can penetrate up to $8-10 \mathrm{~mm}$ depth, $33 \mathrm{MHz}$ up to 6-8 $\mathrm{mm}$, and $75 \mathrm{MHz}$ up to $3-4 \mathrm{~mm}$.

One of the main advantages of HFUS is the visualization with an option of direct internal skin structure measurements (Figure 4), and the detection of micro-morphological changes in different skin pathologies, including skin neoplasms (Figure 5). Such detailed information would not be available to obtain during external examination with palpation, even with dermoscopic optical imaging. Another advantage of skin HFUS is the fact that the obtained US images of the skin represent skin pathology images, therefore, they can be easily interpreted by dermatologists and oncologists. Figure 6 shows HFUS scan taken with $75 \mathrm{MHz}$ probe of a psoriatic papule, with corresponding histological image of the same lesion. This skin lesion was surgically excised, and post-operative pathological measurements matched those taken at HFUS. Figure 6 consist of 2 parts: left part HFUS $75 \mathrm{MHz}$ image and right part histological image with indication of the epidermis, papillary dermis, and reticular dermis as well as hair follicles. Dermal papillae (indicated as " $\mathrm{pd}$ ") in Figure 6 are shown with red arrows. At HFUS and histology images, dermal papillae looks very similar.

The use of HFUS has been studied in normal skin and in various skin pathologies. It has been found useful in differentiation diagnosis of infiltrative (perivascular) and exudative inflammation, hypertrophic or normotrophic scars, keloids, and in skin atrophy. Also, it has been used to support diagnosis and management of skin tumors (Figure 5).

Currently, HFSUs are applied within the following areas: - micro-anatomical structure of healthy skin and echo-graphic signs of aging study;

- assessment of skin condition and anatomical features before cosmetic and plastic surgery, e.g., in Botox or fillers injections and for post-procedure follow-up, such as efficacy and side effects of the procedure, and location of fillers;

- support of differential diagnosis and management in various skin pathologies, including pre-biopsy assessment;

- follow-up after pharmacotherapy, topical therapy, physiotherapeutic, and destructive skin treatments used in dermatology, cosmetology, oncology, and plastic surgery;

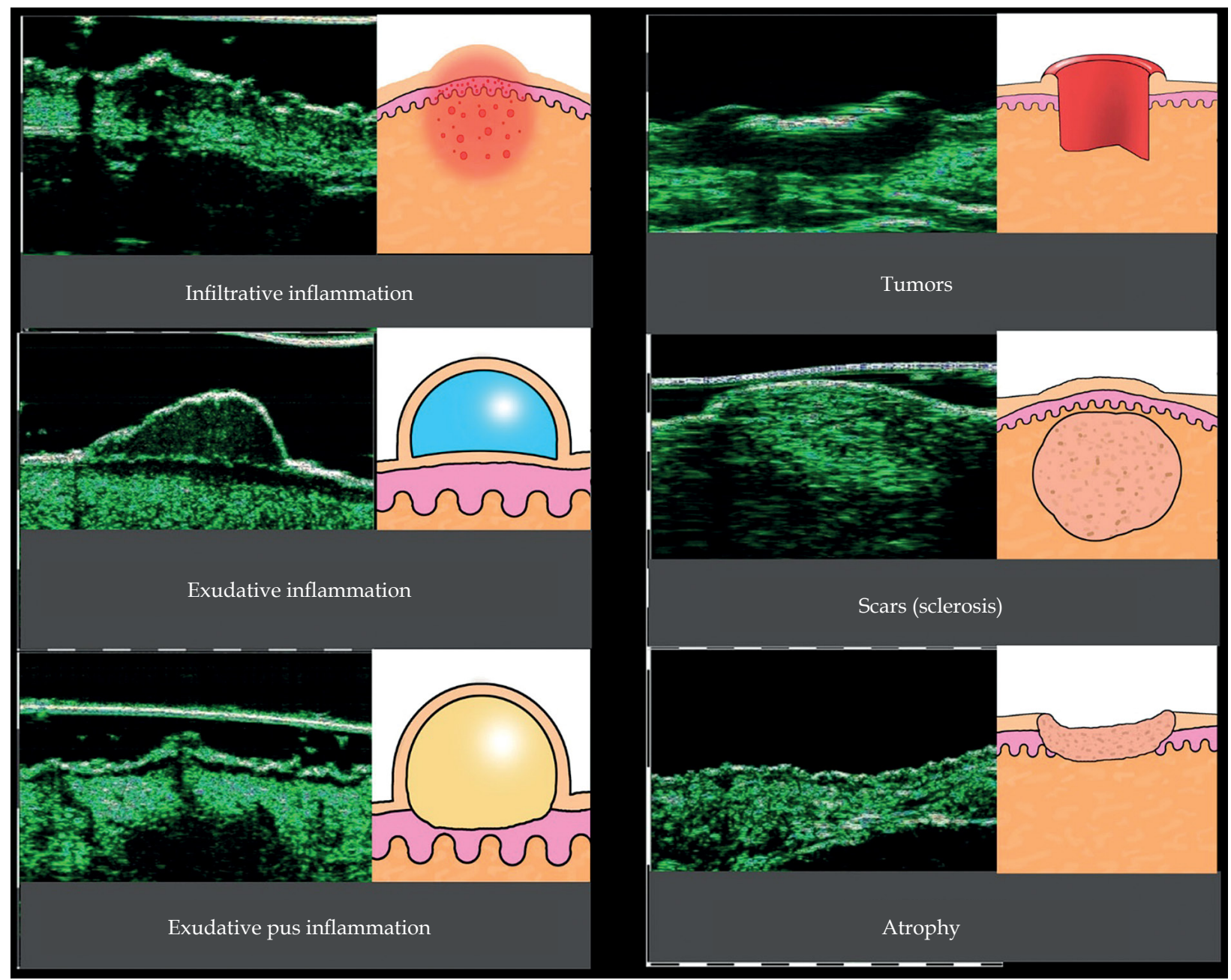

Fig. 5. HFUS images of different skin pathologies 


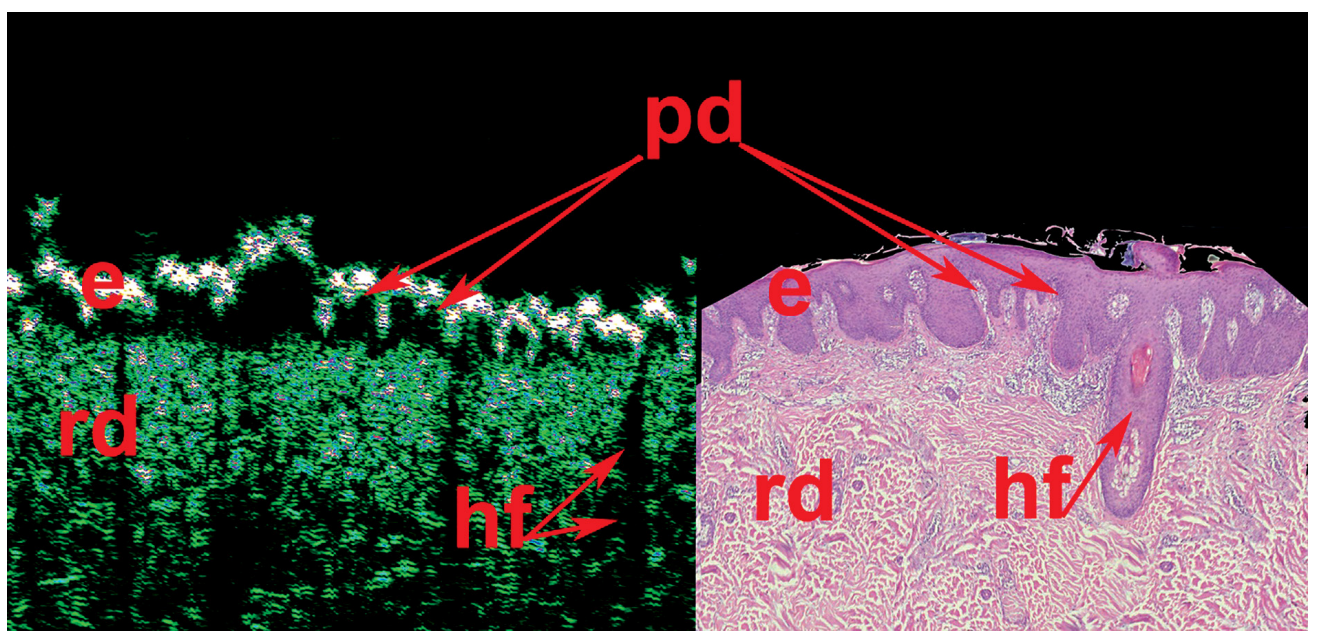

Fig. 6. HFUS scan of a psoriatic papule, with corresponding post-surgical pathology image. e - epidermis, pd - papillary dermis, rd - reticular dermis, hf - hair follicle

- assessment of skin tumors' characteristics, and margins and vascular pattern in dermato-oncology.

\section{The use of HFUS in dermato-oncology}

Non-melanoma skin cancers (NMSC) are the most common malignant skin tumors with basal cell carcinoma (BCC), followed by squamous skin carcinoma (SCC) [7]. The aim of NMSC treatment is to remove the tumor completely with good cosmetic and functional effects [8]. The treatment choice is multifactorial. It depends on the lesion size, including the invasion depth, anatomical location, and clinical type of the tumor [9-11]. These parameters can be successfully assessed with HFUS skin imaging [6, 12-14].

In dermato-oncology HFUS can help with the following information:

- tumor measurements and depth/level of invasion;

- tumor contours and possible peritumoral infiltration;

- guidance for skin biopsy;

- clinical features indicating possible sub-type of NMSC;

- support of optimal management and choice of treatment, e.g., non-invasive versus invasive;

- post-treatment follow-up and diagnosis of early recurrences.

There are multiple studies in various skin pathologies comparing measurements taken during HFUS with those provided at pathological examination. Lassau et al. found a strong correlation between $20 \mathrm{MHz}$ HFUS and pathology in measurements of melanoma thickness [15]. Guitera et al. described a high correlation of HFUS and histological Breslow thickness, with $p<0.001$ [16]. Their measurements were highly reliable also for invasive melanoma and even in the presence of lymphocytic infiltration. Bobadilla et al. used HFUS instead of pre-surgical biopsy, and found similar high correlation in measurements of BCC thickness [17]. Some centers also use HFUS to guide their surgical management in order to assess not only the lesion itself, but also layers of involvement, vascularity patterns, and possible microsatellites, all by the means of non-invasive imaging [18]. HFUS has been also used to monitor treatment response, with some authors indicating a reduction in need for surgical removal of skin lesions post-topical treatment [19].

Various studies demonstrated the use of HFUS in differentiation of clinical forms of BCC. Wortsman et al. retrospectively analyzed HFUS of primary BCC tumors and compared with pathological outcomes [20]. The authors noted a statistically significant association between the number of hyperechoic spots in HFUS and histological sub-types of BCC. Higher hyperechoic spots count was found in the recurrence-prone micronodular, sclerosing variant, and morpheiform BCC sub-types. The presence and count of hyperechoic spots in BCC are considered as risks for a local BCC recurrence. Ultrasonographic features of superficial and nodular basal cell carcinoma were also analyzed and described [12-14, 20]. Below, there are examples of various BCC sub-types assessed by HFUS (Figures 7, 8)

HFUS can be also used in combination with video-dermoscopy in order to increase the accuracy of diagnosis [21]. Video-dermoscopy can help with clinical differentiation between benign and malignant skin tumours, while HFUS evaluates tumour margins and depth of invasion. Figures 9-11 show HFUS with correlating video-dermoscopy images in various BCC sub-types.

In skin oncology clinics, HFUS can provide essential information on the tumor size and margins, particularly the depth of invasion. Such information are crucial in deciding on the form of treatment (such as photodynamic therapy, external beam radiotherapy, or brachytherapy), CT-based planning treatment (e.g., superficial brachytherapy), and in skin monitoring after treatment, in addition to clinical examination [22]. Figure 12 demonstrates HFUS of BCC skin lesion before and after photo-dynamic treatment.

Figure 13 shows HFUS $22 \mathrm{MHz}$ nodular ulcerated BCC scan before radiotherapy and 6 months after finishing the course of radiotherapy treatment. The tumor was visualized as oval-shaped hypo-anechoic area with clear 

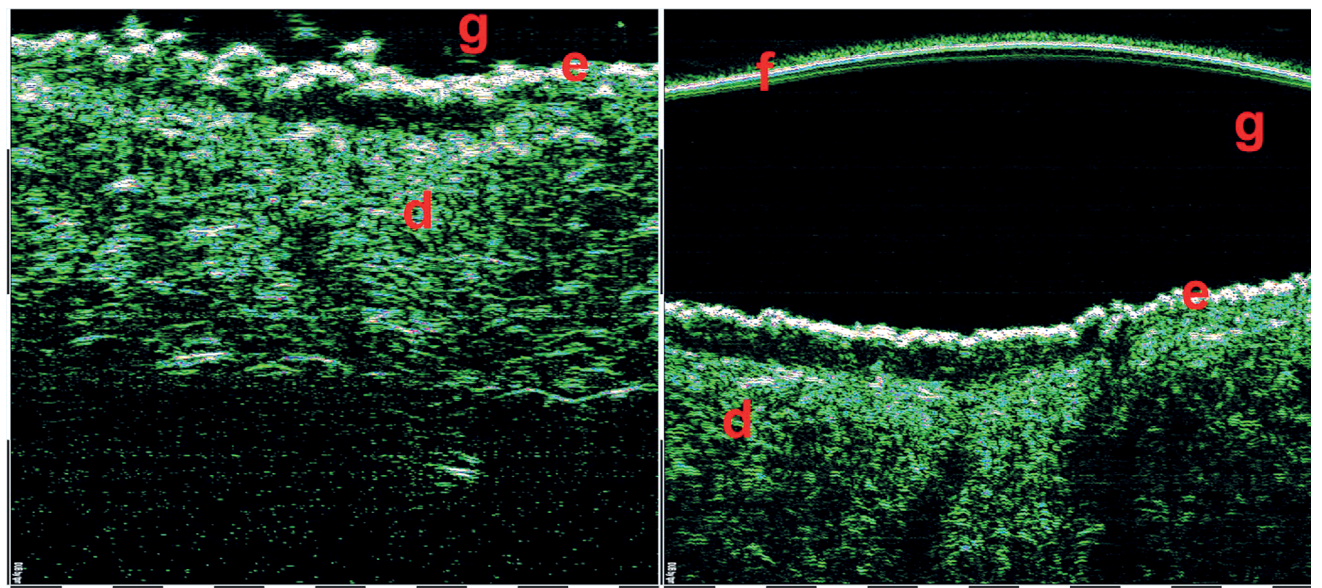

Fig. 7. Superficial basal cell carcinomas (BCCs) $75 \mathrm{MHz}$ HFUS. The hypo- and hetero-echoic areas of elongated strip-shape form located under epidermis, with distinct lateral and lower margins. $\mathrm{f}$ - foil on the probe tip, g - gel, e - epidermis, $\mathrm{d}$ - dermis
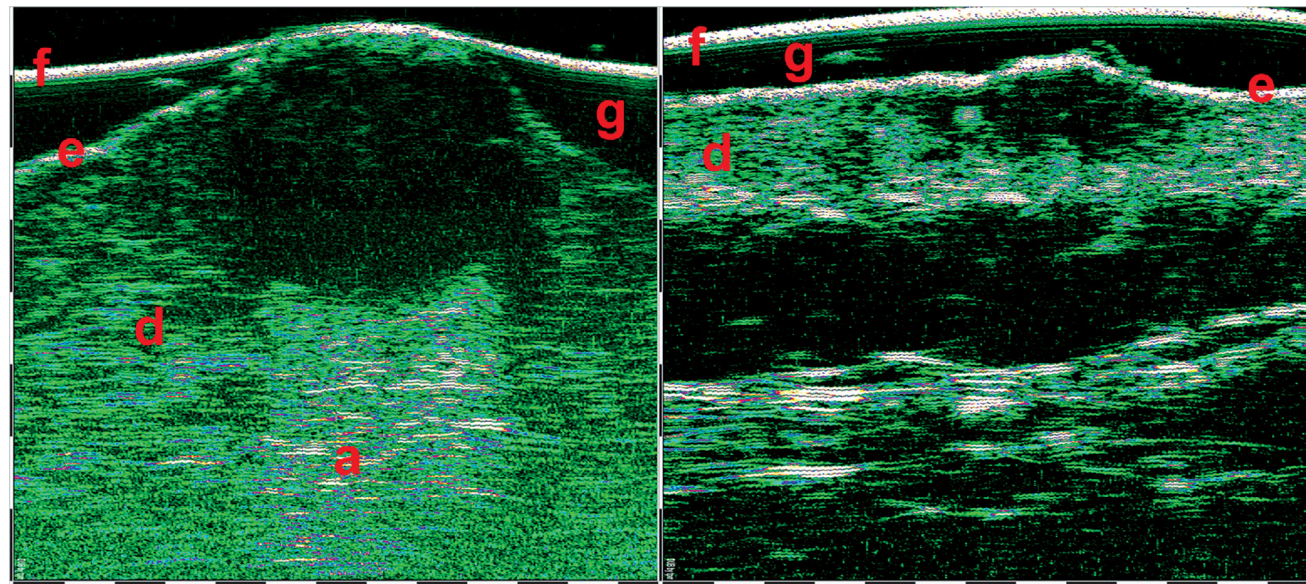

Fig. 8. Nodular basal cell carcinomas (BCCs) $33 \mathrm{MHz}$ HFUS scans. The hypo- and anechoic zones of oval shape, with some hyper-echoic points (on the right scan) visible within the structure. $\mathrm{f}$ - foil on the probe tip, $\mathrm{g}$ - gel, $\mathrm{e}$ - epidermis, $\mathrm{d}$ - dermis. Nodular BCCs have much bigger size and depth, therefore $33 \mathrm{MHz}$ range was used for visualization
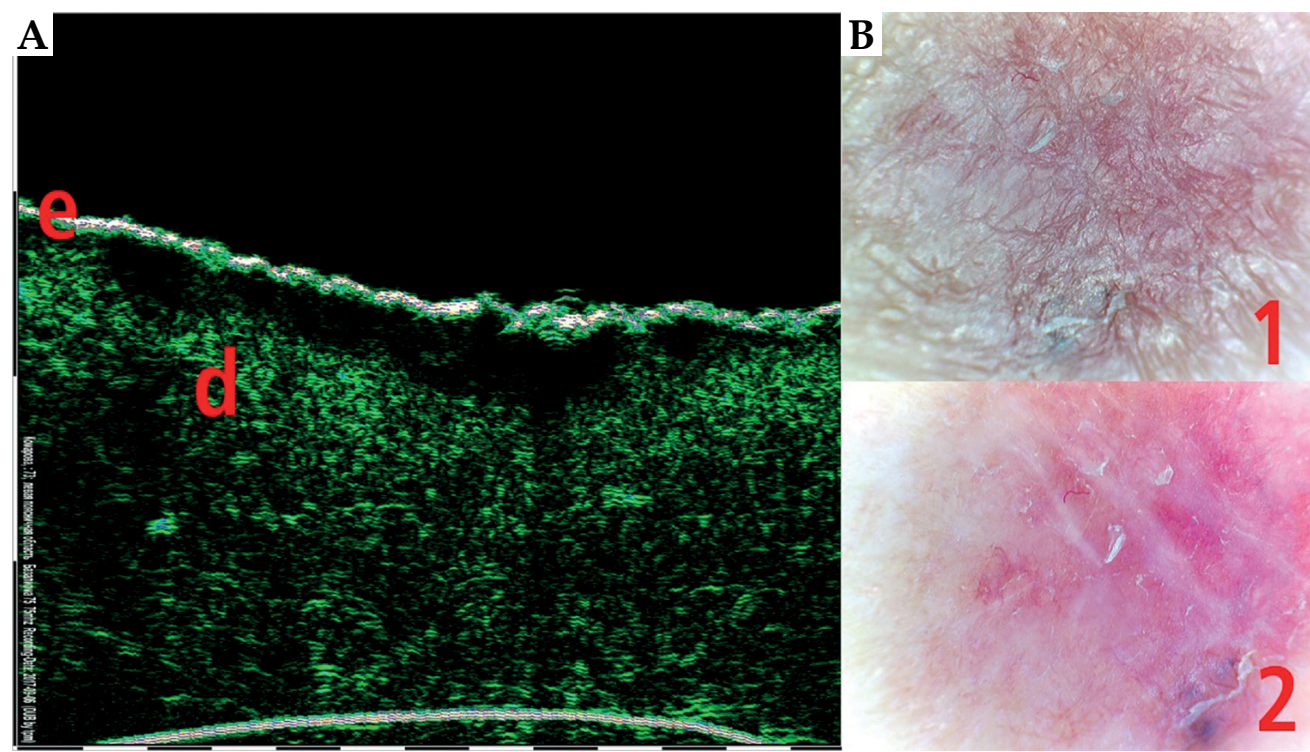

Fig. 9. Superficial basal cell carcinoma (BCC) $75 \mathrm{MHz}$ HFUS scan (A) and video-dermoscopy (B). e - epidermis, d - dermis, 1 - video-dermoscopy image in normal light, 2 - video-dermoscopy image in polarized light 

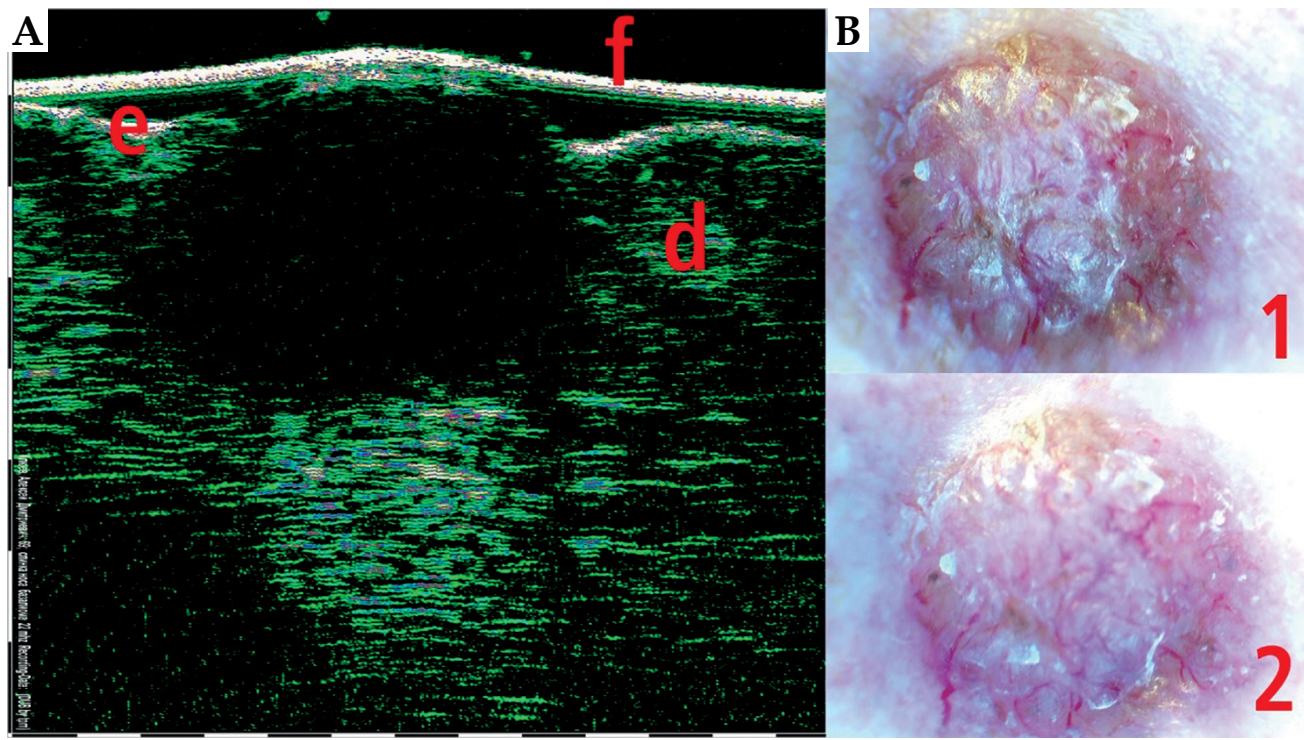

Fig. 10. Nodular basal cell carcinoma (BCC) $33 \mathrm{MHz}$ HFUS scan (A) and video-dermoscopy (B). e - epidermis, d - dermis, $\mathrm{f}$ - foil on the probe tip, 1 - video-dermoscopy image in normal light, 2 - video-dermoscopy image in polarized light
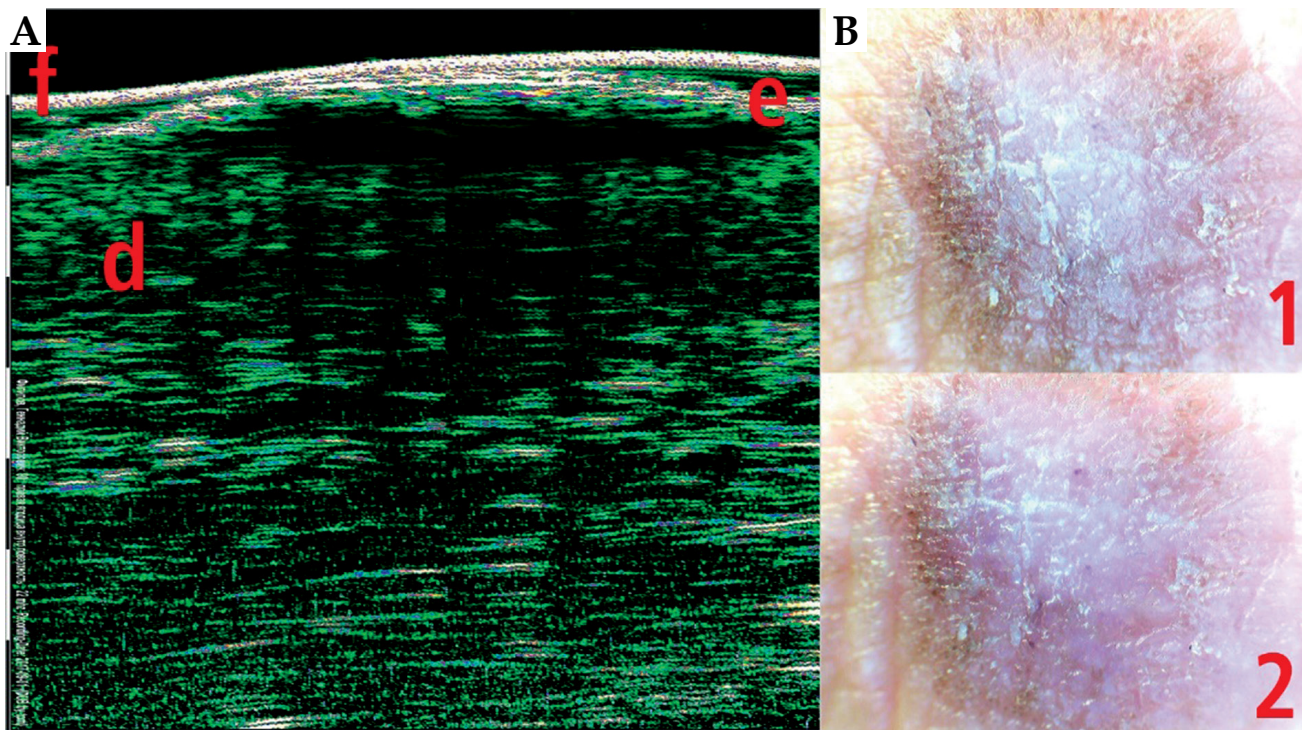

Fig. 11. Skin squamous skin carcinoma (SCC) 33 MHz HFUS scan (A) and video-dermoscopy (B). e - epidermis, d - dermis, $\mathrm{f}$ - foil on the probe tip, 1 - video-dermoscopy image in normal light, 2 - video-dermoscopy image in polarized light

lower and lateral margins, located in the dermis, with invasion depth of $1,670 \mu$, including deep reticular dermis. After radiotherapy, the scarring, dermis, and soft tissues atrophy were visualized. Dermis thickness decreased to $1,140 \mu(1,680 \mu$ in contralateral intact area), superficial sub-dermal fat layer also thinned to $400 \mu(900 \mu$ in contralateral intact area), and fascia pulled up in comparison with the contralateral intact area.

\section{Summary}

HFUS is a feasible and non-invasive imaging modality used to assess normal skin and its pathologies, including skin neoplasms. HFUS cannot replace histology but can potentially be used as an effective ad- ditional diagnostic tool in dermato-oncology. The tumor size, invasion depth, and margins character are the critical parameters for a surgery, radiotherapy, and brachytherapy planning. HFUS is used to identify high recurrence risk of basal cell carcinomas, which are important for the proper treatment choice. HFUS can also help clinicians to assess treatment response, and potentially identify an early recurrence before it can be detected during clinical examination; therefore, an earlier salvage treatment may be considered. HFUS has certain limitations, since this imaging as other US exams, is operator-dependent, and requires specific training and experience. Further studies are needed in order to fully understand the value of HFUS in skin radiotherapy and brachytherapy. 


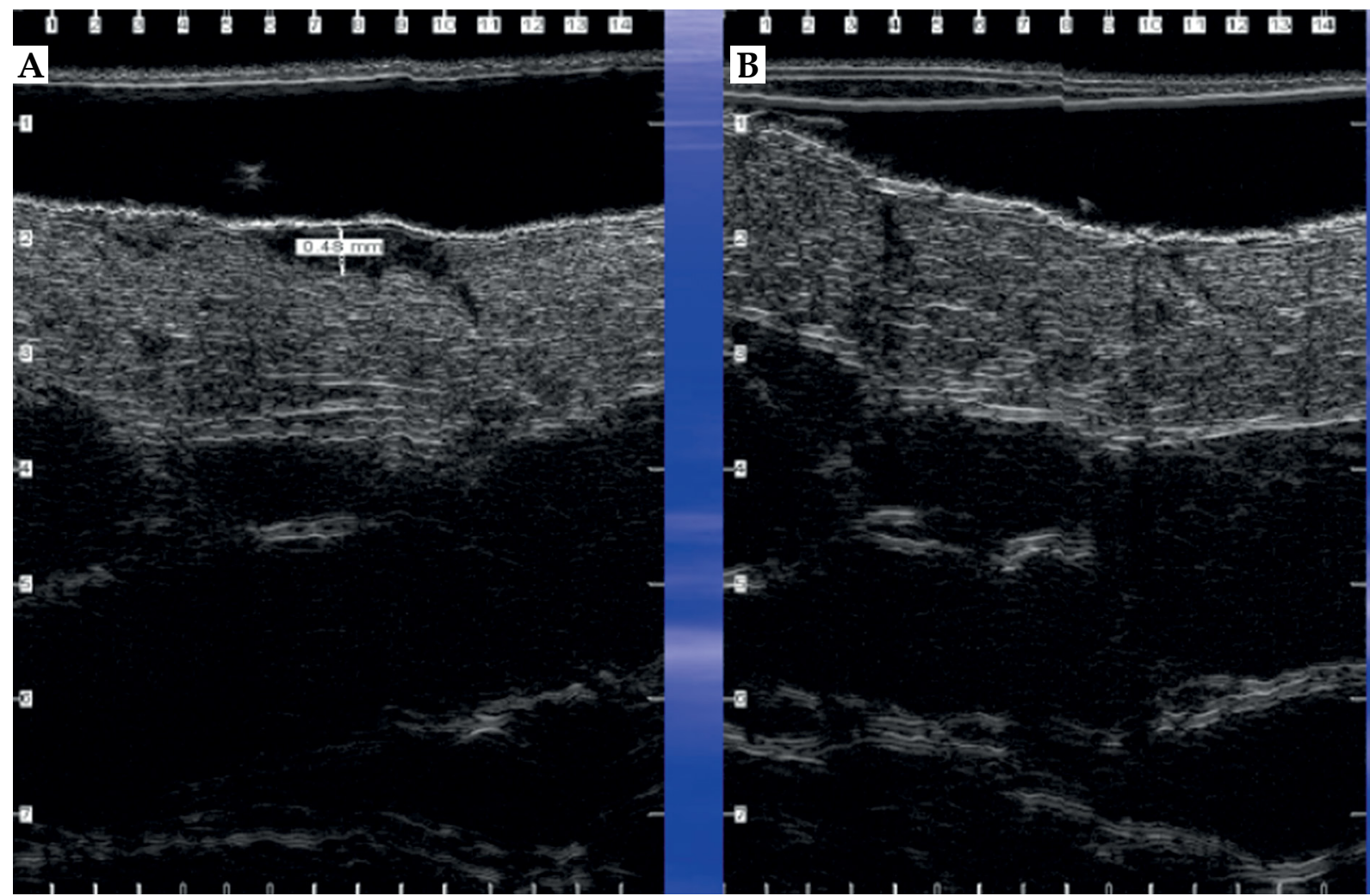

Fig. 12. Skin basal cell carcinoma (BCC) HFUS scan before (A) and after (B) photo-dynamic treatment (curtesy of Mrs. Laura Foster, The Christie NHS Foundation Trust, Manchester, UK)

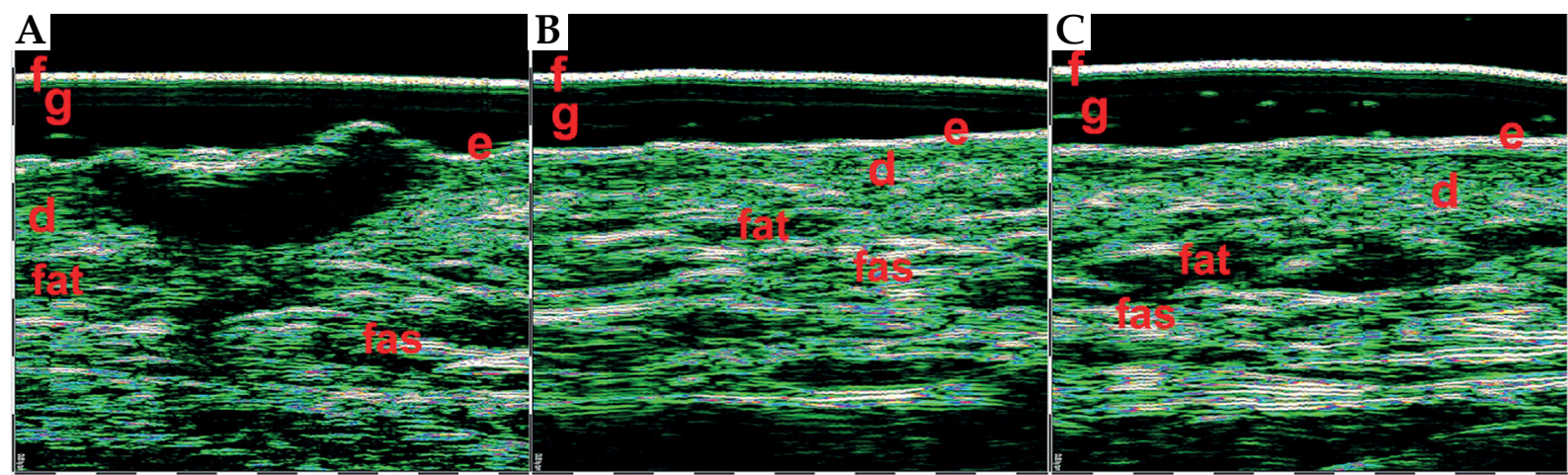

Fig. 13. $22 \mathrm{MHz}$ HFUS scans. Nodular basal cell carcinoma (BCC) before radiotherapy treatment course (A), 6 months after treatment $(\mathbf{B})$, and intact skin in the contralateral anatomic area $(C)$. $f$ - foil on the probe tip, $g$ - gel, e - epidermis, $d$ - dermis, fat - fat tissue, fas - fascia

\section{Disclosure}

The authors report no conflict of interest.

\section{References}

1. Klibanov AL, Hossack JA. Ultrasound in radiology: from anatomic, functional, molecular imaging to drug delivery and image-guided therapy. Invest Radiol 2015; 50: 657-670.

2. Szabo T. Diagnostic ultrasound imaging: inside out. $2^{\text {nd }} \mathrm{ed}$. Elsevier 2013; 23-26.

3. Oltulu P, Ince B, Kokbudak $\mathrm{N}$ et al. Measurement of epidermis, dermis, and total skin thicknesses from six different body regions with a new ethical histometric technique. Turk J Plast Surg 2018; 26: 56-61.
4. Maiti R, Duan M, Danby SG et al. Morphological parametric mapping of 21 skin sites throughout the body using optical coherence tomography. J Mech Behav Biomed Mater 2020; 102: 103501.

5. Nedelec B, Forget NJ, Hurtubise T et al. Skin characteristics: normative data for elasticity, erythema, melanin, and thickness at 16 different anatomical locations. Skin Res Technol 2016; 22: 263-275.

6. Mlosek RK, Migda B, Migda M. High-frequency ultrasound in the 21st century. J Ultrason 2021; 20: e233-e241.

7. Rogers HW, Weinstock MA, Harris AR et al. Incidence estimate of nonmelanoma skin cancer in the United States, 2006. Arch Dermatol 2010; 46: 283-287.

8. Marzuka AG, Book SE. Basal cell carcinoma: Pathogenesis, epidemiology, clinical features, diagnosis, histopathology, and management. Yale J Biol Med 2015; 88: 167-179. 
9. Bichakjian CK, Olencki T, Aasi SZ et al. Basal cell skin cancer, Version 1.2016, NCCN Clinical Practice Guidelines in Oncology. J Natl Compr Cancer Netw 2016; 14: 574-597.

10. Telfer NR, Colver GB, Morton CA at al. British Association of Dermatologists. Guidelines for the management of basal cell carcinoma. Br J Dermatol 2008; 159: 35-48.

11. Trakatelli M, Morton C, Nagore E et al. Update of the European guidelines for basal cell carcinoma management. Eur J Dermatol 2014; 24: 312-329.

12. Wortsman X. Sonography of facial cutaneous basal cell carcinoma. J Ultrasound Med 2013; 32: 567-572.

13. Khlebnikova A, Molochkov V, Selezneva E et al. Ultrasonographic features of superficial and nodular basal cell carcinoma. Med Ultrason 2018; 20: 475-479.

14. Khlebnikova A, Molochkov V, Selezneva E et al. Basal cell carcinoma invasion depth determined with 30 and $75 \mathrm{MHz}$ high-frequency ultrasound and histopathology - a comparative study. Med Ultrason 2020; 22: 31-36.

15. Lassau N, Spatz A, Lavril MF et al. Value of high frequency US for preoperative assessment of skin tumors. Radiographic 1997; 17: 1559-1565.

16. Guitera P, Li LX, Crotty $\mathrm{K}$ et al. Melanoma histological Breslow thickness predicted by $75-\mathrm{MHz}$ ultrasonography. Br J Dermatol 2008; 159: 364-369.

17. Bobadilla F, Wortsman X, Munoz C et al. Pre-surgical high resolution ultrasound of facial basal cell carcinoma: correlation with histology. Cancer Imaging 2008; 8: 163-172.

18. Marmur ES, Berkowitz EZ, Fuchs BS et al. Use of high-frequency, high-resolution ultrasound before Mohs surgery. Dermatol Surg 2010; 36: 841-847.

19. Bhatta AK, Keyal U, Liu Y. Application of high frequency ultrasound in dermatology. Discov Med 2018; 26: 237-242.

20. Worthman X, Vergara P, Castro F et al. Ultrasound as predictor of histologic subtypes linked to recurrence in basal cell carcinoma of the skin. J Eur Acad Dermatol Venereol 2014; 29: 702-707.

21. Pellacani G, Seidenari S. Preoperative melanoma thickness determination by $20-\mathrm{MHz}$ sonography and digital videomicroscopy in combination. Arch Dermatol 2003; 139: 293-298.

22. Veness MJ, Delishaj D, Barnes EA et al. Current role of radiotherapy in non-melanoma skin sancer. Clin Oncol (R Coll Radiol) 2019; 31: 749-758. 